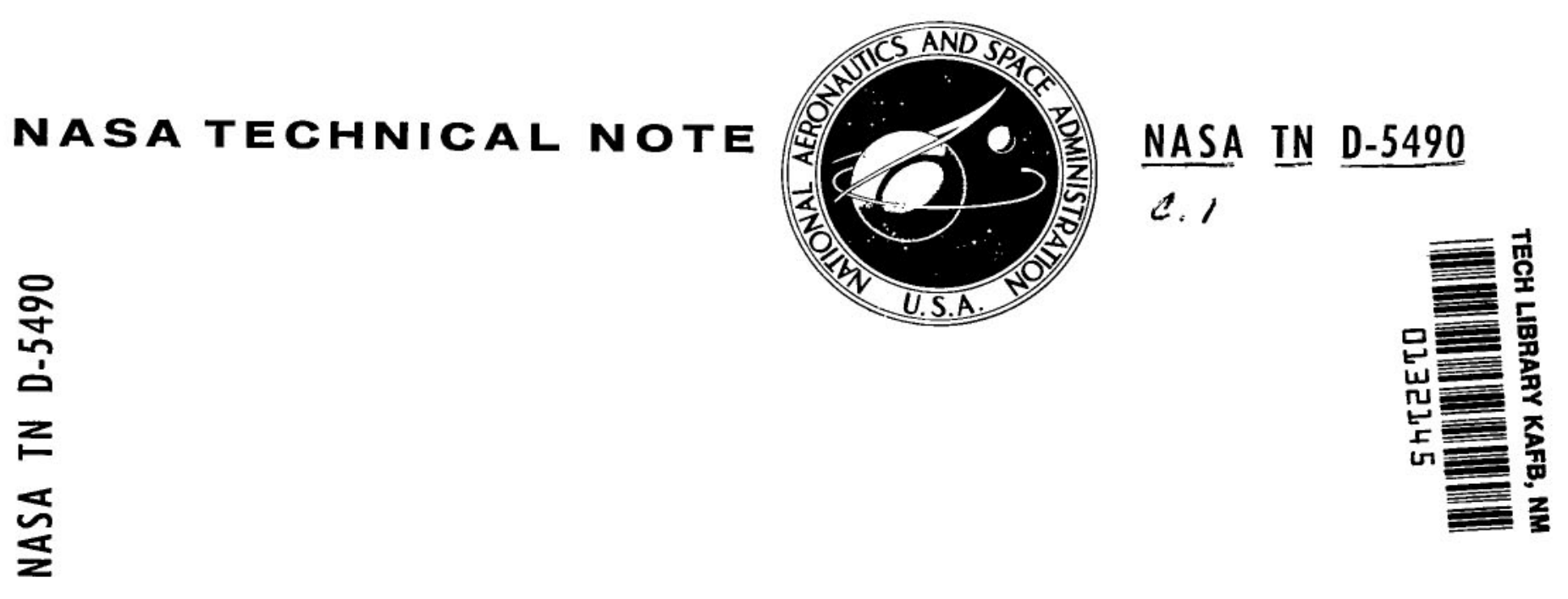

LOAN COPY: RETURA TO

AFWL (WLOL-2)

KIRTLAND AFB, N MEX

\title{
ENERGY DISTRIBUTION OF A SPIRALING ELECTRON BEAM
}

by Bernhard Kulke

Electronics Research Center

Cambridge, Mass.

NATIONAL AERONAUTICS AND SPACE ADMINISTRATION - WASHINGTON, D. C. - NOVEMBER 1969 


\begin{tabular}{|l|l|}
\hline 1. Report No. & 2. Government Access \\
NASA TN D-5490 & \\
4. Titlo and Subtitle & \\
Energy Distribution of a \\
Spiraling Electron Beam \\
7. Author(s) \\
Bernhard Kulke \\
9. Performing Orgonization Name and Address \\
Electronics Research Center \\
Cambridge, Mass.
\end{tabular}

12. Sponsoring Agency Name and Address

National Aeronautics and Space Administration
3. Recipient's Catalog No.

5. Report Date

November 1969

6. Porforming Organization Code

8. Porforming Organization Report No. $\mathrm{C}-97$

10. Work Unit No.

129-02-21-02-25

11. Controct or Gront No.

13. Type of Report and Period Covered

Technical Note

14. Sponsoring Agency Code

15. Supplementary Notes

16. Abstract

A retarding-field analyzer was used to investigate the energy conversion and the energy spread of a spiraling electron beam, where the trajectory is generated from a magnetic corkscrew-magnetic ramp combination. It was found that in the absence of spacecharge effects, a relatively narrow, near-rectangular energy distribution will be generated by the corkscrew when the axial magnetic field is tuned to cyclotron resonance. The normalized width of this distribution is approximately independent of the degree of energy conversion, in agreement with the theory, and the mean transverse energy varies as the square of the corkscrew current. The magnetic ramp amplifies both the mean transverse energy and the energy spread linearly. Both these quantities also can be estimated by using the mirroring effect of the magnetic ramp, in conjunction with variable corkscrew current.

17. Koy Words

- Retarding-Field Analyzer

- Spiraling Electron Beam

- Magnetic Corkscrew-Magnetic Ramp Combination

- Variable Corkscrew Current
18. Distribution Statement

$$
\text { Unclassified - Unlimited }
$$

19. Security Classif. (of this report)

20. Security Classif. (of this page)

Unclassified

Unclassified

\begin{tabular}{|c|c|}
\hline 21. No. of Pages & 22. Price* \\
22 & $\$ 3.00$
\end{tabular}

*For sale by the Clearinghouse for Federal Scientific and Technical Informatıon Springfield, Virginia 22151 


\title{
ENERGY DISTRIBUTION OF A SPIRALING ELECTRON BEAM
}

\author{
By Bernhard Kulke \\ Electronics Research Center
}

\section{SUMMARY}

Complementary to an in-house project concerned with spiralingbeam oscillators suitable for millimeter-wave generation, a retarding-field analyzer was used to investigate the energy conversion, and especially the energy spread, incurred when the electron beam trajectory was generated during passage through a magnetic corkscrew followed by a magnetic ramp. It was found that in the absence of space-charge effects, a relatively narrow, near-rectangular energy distribution was generated by the corkscrew when the axial magnetic field was tuned to cyclotron resonance. The normalized width of this distribution is approximately independent of the degree of energy conversion, in agreement with the theory, and the mean transverse (converted) energy varies as the square of the corkscrew current. The magnetic ramp amplifies both the mean transverse energy and the energy spread linearly, as expected from the adiabatic assumption. Both quantities also can be estimated by using the mirroring effect of the magnetic ramp, in conjunction with variable corkscrew current.

\section{INTRODUCTION}

In a prior report (ref. 1) an in-house project at ERC has been described that is concerned with spiraling-beam oscillators where the beam trajectory is generated during passage through a cork.. screw-mirror system, i.e., a magnetic corkscrew (ref. 2) followed by a magnetic ramp. This type of device has demonstrated some capability and holds further promise as a high-power millimeterwave source. RF power is extracted from the spiraling electron cloud by means of a simple smooth waveguide or cavity, and one thus avoids the usual difficulty both of machining an ultraprecise slow-wave structure and of forming and steering a highdensity linear electron beam close to such a structure. During work on a prototype oscillator which was built and tested at ERC (ref. 3), it became evident that probably the most important limitation on the available power and the efficiency of this type of device was the finite energy spread that arises when the axial momentum of the initially monoenergetic electron beam is converted into transverse (orbital) momentum by the combined action of the corkscrew and the magnetic ramp. The rf interaction can take place only over a finite range of axial velocities. and all electrons that are not in this range are thus lost to the interaction. In order to get some idea of the magnitude of the 
total energy spread and of the tradeoffs involved in minimizing it, a retarding-field velocity analyzer of a type described by Caulton (ref. 4) was built that responded selectively to the axial momentum of the beam electrons, independent of their orbital velocity. This report will describe the latter device, the data it yielded, and some conclusions that are suggested by these data. Both the measurements and their interpretation are aimed at spiraling-beam oscillators, and they are rather different in this sense from earlier work (refs. 5,6, and 7) which was motivated originally by the potential use of a magnetic corkscrew as a component in a magnetic mirror system for plasma confinement, i.e., in nuclear fusion experiments.

\begin{tabular}{|c|c|}
\hline & SYMBOLS* \\
\hline $\mathrm{v}_{\mathrm{O}}$ & DC beam potential at gun \\
\hline $\mathrm{v}_{\text {ret }}$ & retarding potential \\
\hline$\Delta \mathrm{V}$ & energy spread \\
\hline $\mathrm{V}_{11}$ & axial beam energy \\
\hline $\mathrm{V}_{\perp}$ & transverse beam energy \\
\hline$\left\langle v_{11}\right\rangle$ & mean axial energy \\
\hline$\left\langle v_{\perp}\right\rangle$ & mean transverse energy \\
\hline$B_{0}$ & value of magnetic field at low plateau \\
\hline $\mathrm{B}_{1}$ & value of magnetic field at high plateau \\
\hline M & mirror ratio, $\quad M=\frac{1}{B_{0}}$ \\
\hline$I_{0}$ & cathode current \\
\hline$I_{\text {cork }}$ & corkscrew current \\
\hline I Iow & low-field current \\
\hline$I_{\mathrm{COll}}$ & target or collector current \\
\hline a (z) & axial velocity \\
\hline
\end{tabular}

*Energies are expressed in eV, with the (e) suppressed. 

$\mathrm{p}(\mathrm{z}) \quad$ corkscrew pitch
$\eta=\frac{e}{m} \quad$ charge-to-mass ratio
K corkscrew (conversion) efficiency

\section{THE EXPERIMENTAL APPARATUS}

The instrument used in this work is shown to scale in Figure 1 , together with a typical axial magnetic field profile in which it was immersed. The electron gun consisted of a $1-\mathrm{mm}$ dia. Philips cathode, a conical focus cup, and an anode with a 1-mm aperture. The Pierce-gun configuration as used really was redundant because the cathode was immersed in a magnetic field of many times the Brillouin value, so that the beam was essentially in confined flow. The full beam voltage of $2-6 \mathrm{kV}$ was applied directly between cathode and anode, and the beam current was controlled by temperature-limiting the cathode. The uniformity of the low plateau of the axial magnetic field was held to within 1 percent over the gun region, including a large part of the magnetic corkscrew. This uniformity was achieved by shielding the low-field solenoid from the high-field electromagnet which would otherwise set up a strong gradient in the low-field region. The shielding consisted of 1/4-inch thick ingot iron plates that were strapped to the low-field coil in a box-like arrangement. No iron was used anywhere else in the magnetic circuit. The spatial ripple in the high-field plateau was caused by the use of three discrete solenoids, the geometry of which unfortunately did not permit Helmholtz-pair spacing. However, the performance of the velocity analyzer turned out to be markedly insensitive to its axial position with respect to these ripples, and their existence was therefore neglected.

The magnetic corkscrew is a device that serves to impart transverse momentum to an axially-flowing electron beam. For a certain range of axial velocities, a typical electron is subject to a periodic force that acts at right angles to the total magnetic field and remains in phase with the transverse velocity component associated with the cyclotron orbit of the electron. The periodic fields thus appear circularly polarized in the reference frame moving axially with the orbiting electron. This results in a cyclotron-resonant transfer of energy from the axial into the transverse direction, while the total energy is conserved.

The corkscrew used here consisted of a $33 \mathrm{~cm}$ long quadrufilar helix that was wound externally around the non-magnetic $5 / 8$ inch O.D. drift tube, using \#14-gauge enameled copper wire, with a constant pitch of $3.33 \mathrm{~cm}$. Typically, this corkscrew was 


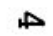

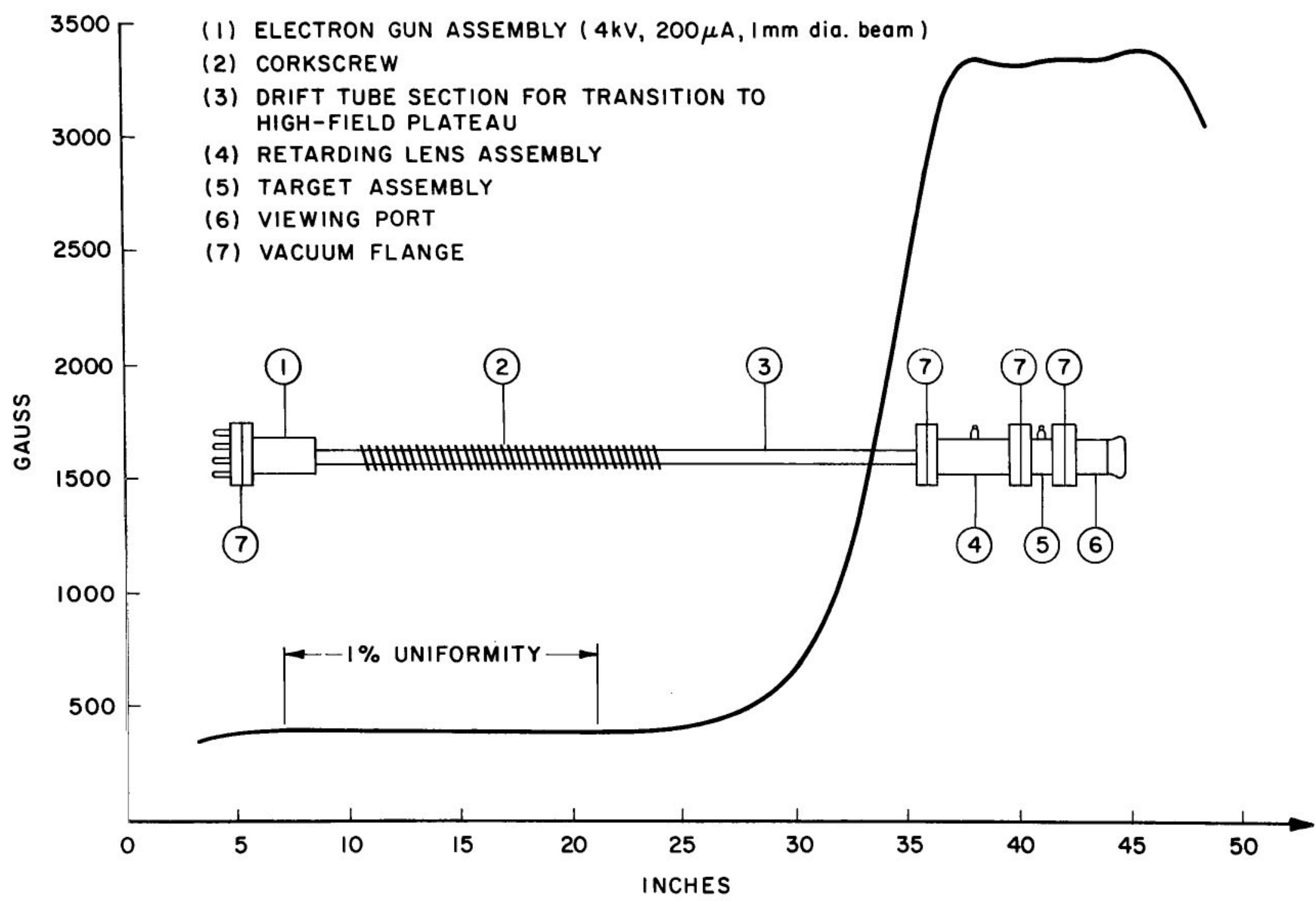

Figure 1. The velocity analyzer, drawn to scale in the axial magnetic field profile 
energized with dc currents of 2-3A, giving a transverse field component of $1-2 \mathrm{G}$ on the beam axis. Assuming a maximum beam diameter of $4 \mathrm{~mm}$, the radial variation of the corkscrew-field over the beam cross section was calculated to be about five percent. Synchronism (resona'nce) of the space-periodic transverse magnetic field with a $4 \mathrm{kV}$ beam was obtained when the axial field was held at 400G. Beams of different energy were accomodated by changing the axial field $B_{0}$ such that

$$
\frac{{ }^{B_{O}}}{\sqrt{\mathrm{v}_{\mathrm{O}}}}=\text { constant. }
$$

While the energy conversion occurs, the axial velocity $u(z)$ decreases and the electron goes out of resonance. Thus, to increase the $Q$ of the system, the period of the field should be decreased with distance in order to be matched to the electron motion. However, for these measurements, the typical change in axial velocity over the corkscrew length was only 3-5 percent, and the corresponding tapered pitch would have been difficult to wind accurately. The corkscrew therefore was wound with constant pitch.

The analyzer head was of a type described by Caulton (ref. 4), and an assembly drawing is shown in Figure 2. The retarding action takes place on or near the axis of the cylindrical retarding lens. The diameter of this lens is made large compared to the spiraling beam diameter so that the equipotential planes encountered by the beam electrons are perfectly normal to the beam axis; it is this feature which assures that the lens controls only the axial component of momentum, but has no effect on the transverse component. To enable accurate centering of the beam, a tungsten-mesh target is mounted behind the retarding lens. This also serves as the collector electrode. The target can be viewed through a glass window, and accurate beam alignment can be achieved by centering the incandescent image of the beam on the target. The entire device is connected to an appendage pump (0.2. liter Vac Ion) both to monitor pressure and to absorb any residual outgassing after the initial bakeout. The demountable construction, using bakeable crushed-copper vacuum flanges, permits easy modification of the device.

\section{MEASURED DATA AND THEIR INTERPRETATION}

The basic measurement performed with the velocity analyzer is an energy analysis. That is, one observes the collector (target)

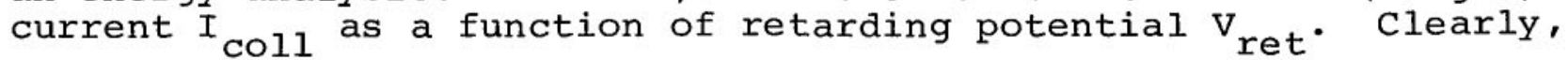
the retarding electric field will cut off those beam electrons 
(1) RETARDING LENS (CYLINDER)

(2) TUNGSTEN MESH TARGET, DIPPED IN COLLOIDAL GRAPHITE SOLUTION

(3) VIEWING TUBE AND TARGET ELECTRODE

(4) GLASS VIEWING PORT

(5) CERAMIC FEEDTHROUGH INSULATORS

(6) VACUUM FLANGES (CRUSHED-COPPER TYPE)

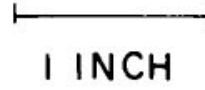

(7) BORON NITRIDE COLLARS

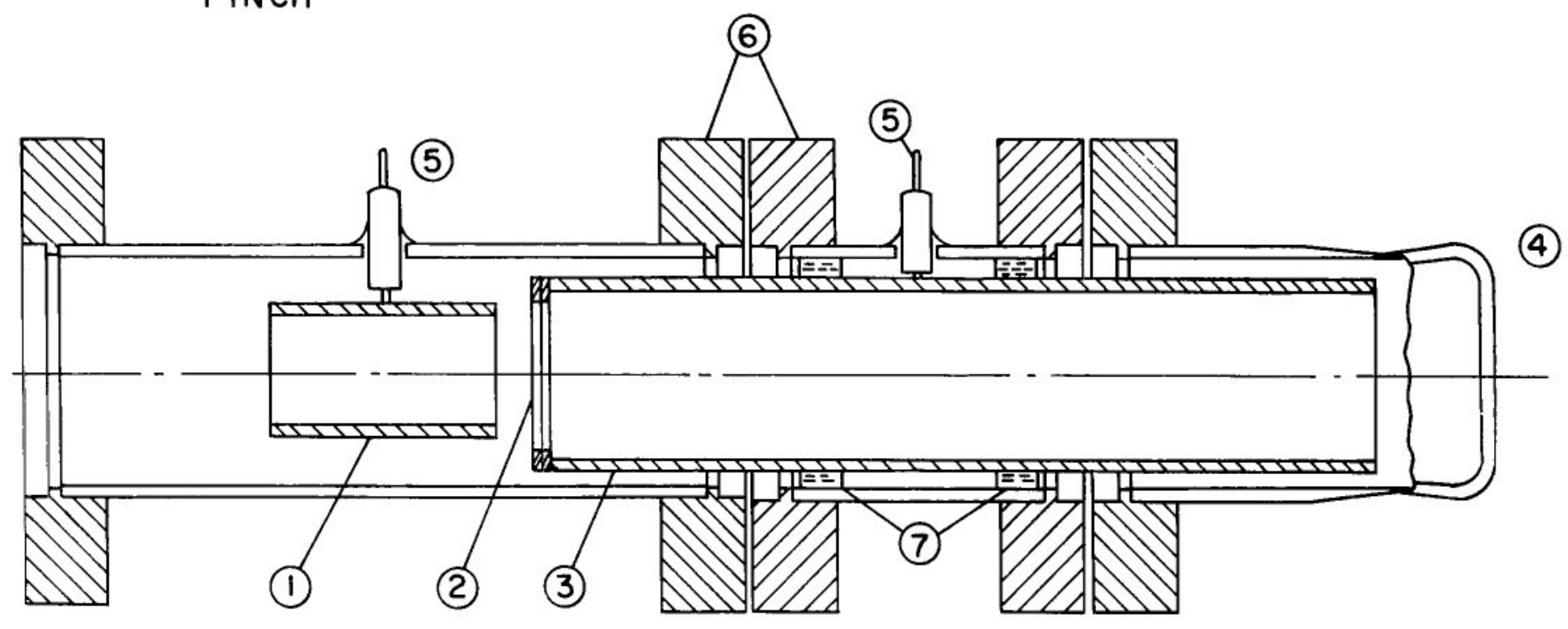

Figure 2. Assembly drawing of the analyzer head 
with axial kinetic energy less than $\mathrm{eV}_{\text {ret }}$ (In the presence of a strong axial magnetic field, there will be backstreaming of the mirrored electrons along the beam, but under conditions of negligible space charge in the forward beam this effect probably can be neglected also). The resultant $I_{\text {coll }}$ cutoff characteristic is then plotted for different combinations of beam parameters, such as beam current $I_{0}$ (space charge), corkscrew current $I_{\text {cork' }}$ mirror ratio $M=\frac{B_{1}}{B_{0}}$, i.e., the ratio of the high to the low plateau of the axial magnetic field, and finally, the beam voltage $\mathrm{V}_{0}$. The results of such measurements will now be described. All measurements were made with the beam pulsed.

\section{Space-Charge Effects}

The range of beam voltages used in these measurements $(2-6 \mathrm{kV})$ was governed by the available retarding voltage supply. As it had previously been found (ref. 4) that space-charge effects in the retarding-lens region could distort the data, it was important to test for such effects in the present series of measurements. The result is shown in Figure 3 , where the beam current was changed by an order of magnitude while $V_{O}, M$ and $I_{\text {cork }}$ were held constant. For the purposes of this paper, the term energy spread $(\Delta V)$ will be used as indicated in Figure 3 , meaning the voltage difference between the point where $I_{\text {coll }}$ first begins to decrease, and where it reaches zero. From Figure $3, \frac{\Delta V}{V_{0}} \simeq 0.05$ regardless

of the beam current, and notwithstanding a slight change in shape of the cutoff characteristic. This result supports an assumption which will be made throughout this paper, i.e., that space-charge effects, either in the retarding-lens region or elsewhere, do not significantly affect the measured energy spread.

The effect of changing $V_{0}$ and the implications of the normalization $\frac{\Delta V}{V_{O}}$ are discussed further below.

\section{Trajectory Modulation in the Corkscrew Region}

The effect of the corkscrew field on the beam is governed by the cyclotron resonance referred to earlier. One tunes the electrons to resonance by adjusting the low-field plateau, as shown in Figure 4, where the target current is plotted vs lowfield amplitude. As the electrons approach resonance, the 


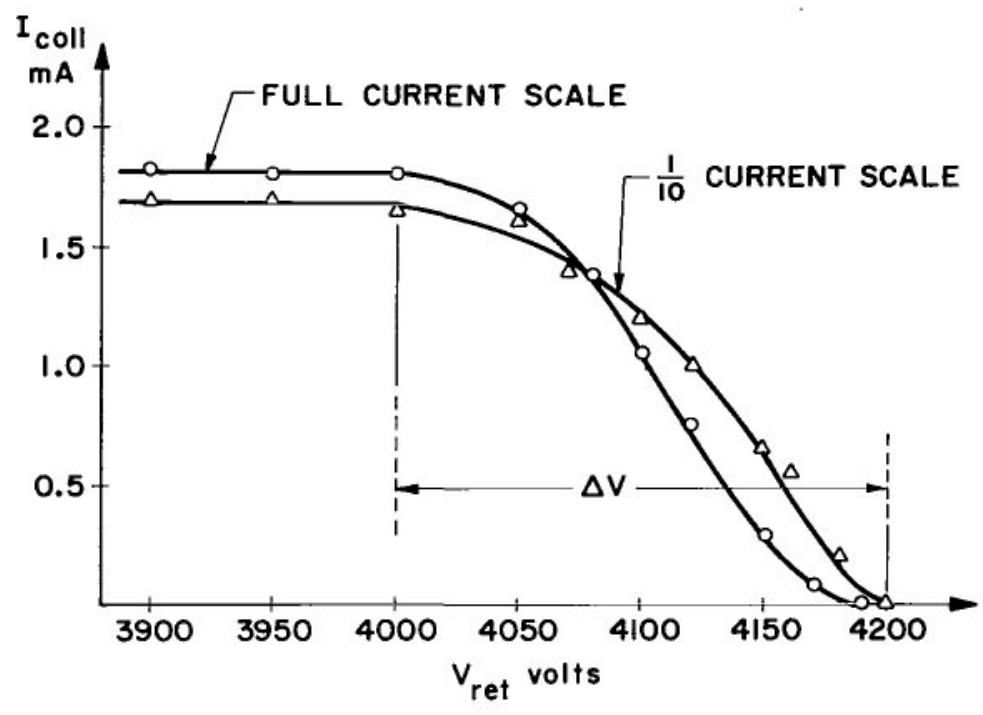

Figure 3. Typical target-current cutoff curves. These curves were plotted for beam currents different by an order of magnitude, in order to investigate the effects of space charge on the measurement. Mirror ratio $M=8.53$ and $I_{\text {cork }}=0$.

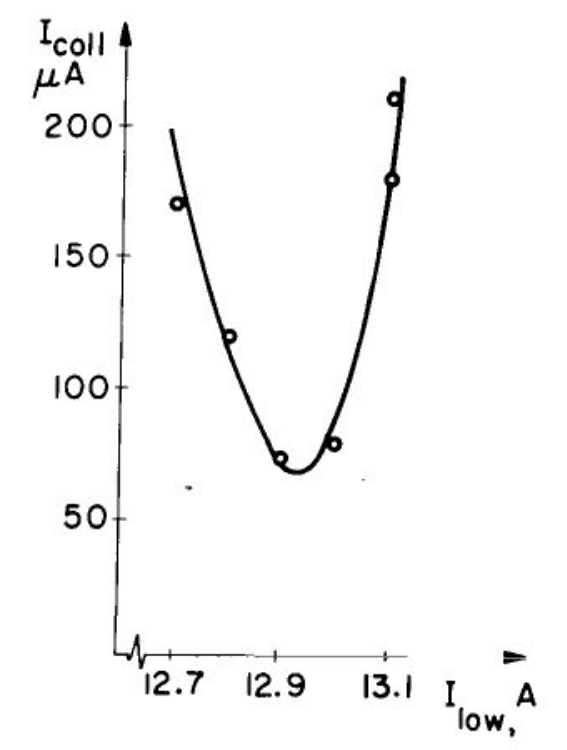

Figure 4. A plot of target current vs low-field current, near cyclotron resonance at $V=4 \mathrm{kV}$. The corkscrew current is $I_{\text {cork }}=2.5 \mathrm{~A}$, and $V_{\text {ret }}=2500 \mathrm{~V}$. The corkscrew was designed to resonate with the beam at $\mathrm{I}_{10 \mathrm{~W}}=13.2 \mathrm{~A}$. 
corkscrew becomes more effective in converting axial into transverse momentum, until finally some of the electrons are left with axial energies below the $2500 \mathrm{~V}$ threshold that is set by the retarding potential, and a drop in I $I_{\text {coll }}$ occurs. However, the measured resonance condition occurs at $12.95 \mathrm{~A}$, or $392 \mathrm{G}$, rather than the design value of 13.2A, or 400G. The physical reason for this is not clear, but it will be seen presently that the best performance of the corkscrew, in terms of minimum energy spread, is indeed obtained near the design resonance.

The typical behavior of the target-current cutoff characteristics when $I_{\text {cork }}$ is changed with $M$ and $V_{0}$ constant, is shown in Figure 5.Increasing the transverse beam modulation (greater $I_{\text {cork }}$ ) results in some increase of axial energy spread, mainly by lengthening the tails of the energy distribution. There also appears to be some "mirroring" (beam reflection before the beam gets to the retarding lens) with $I_{\text {cork }}=3.5 \mathrm{~A}$. However, a decrease of target current is also observed for $I_{\text {cork }}=0$ with $V_{\text {ret }} \leq 700$ volts, and the observed "mirroring" may actually be the same phenomenon, which could be due to the emission and subsequent backstreaming of secondaries from the tungsten and graphite target surface. This type of behavior was noted consistently, and for this reason measurements were not taken, in general, in the range $\mathrm{V}_{\text {ret }}<700 \mathrm{~V}$.

Cutoff characteristics of the type shown in Figure 5 can be converted directly to velocity distribution curves by simple differentiation, as follows. If an electron beam has the (axial) velocity distribution $f(u)$ and this is truncated at some velocity $u_{\text {ret' }}$ corresponding to a retarding potential $v_{\text {ret' }}$ then the remaining beam current is given by

$$
I_{\text {coll }}=e \int_{u_{\text {ret }}}^{\infty} u f(u) d u
$$

where $f(u)$ is normalized such that the total number of electrons per unit of beam volume is

$$
\begin{aligned}
& N_{e} \triangleq \int_{0}^{\infty} f(u) d u \\
& u=\sqrt{\frac{2 e V_{\mathrm{II}}}{m}}
\end{aligned}
$$




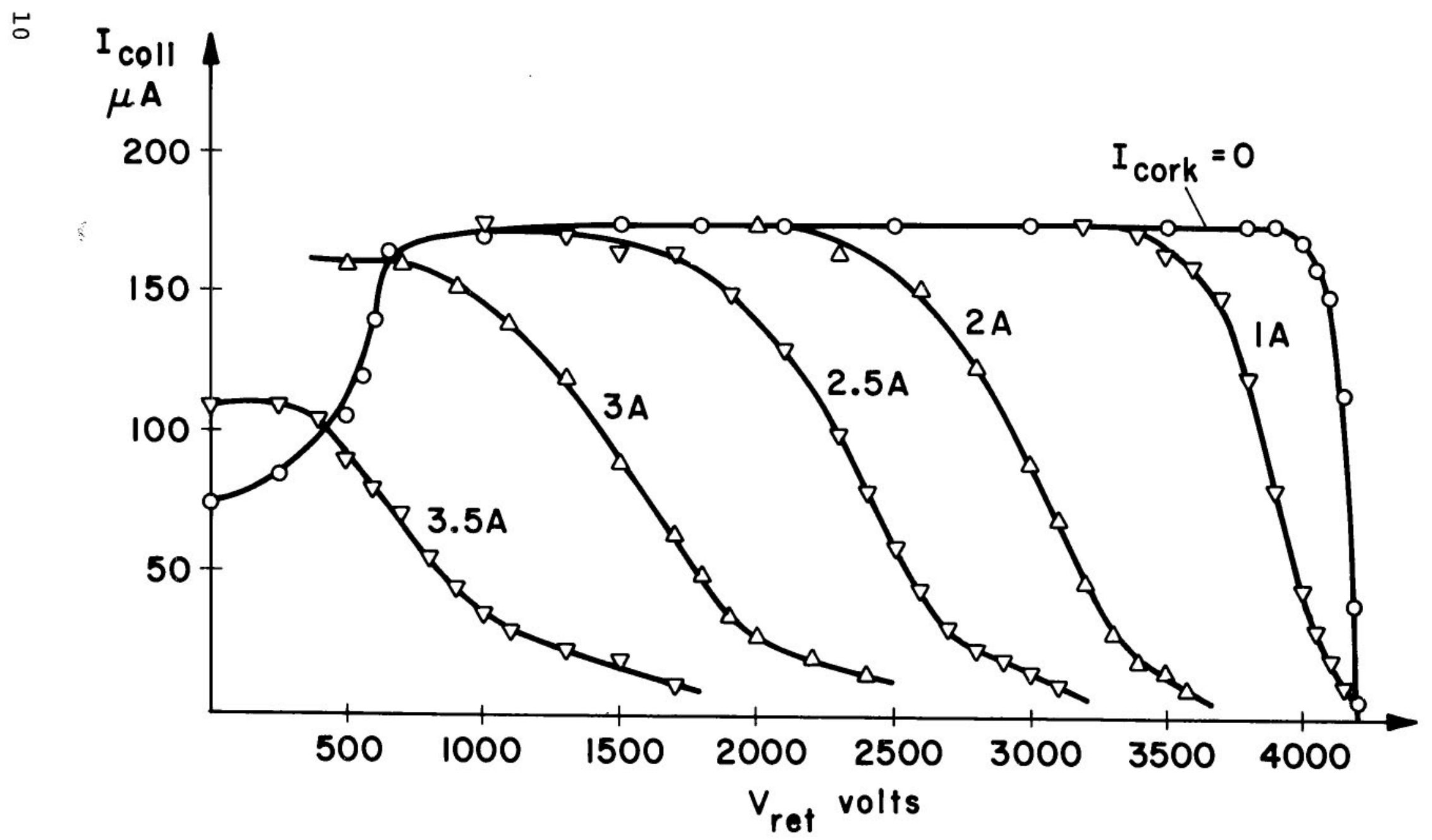

Figure 5. A typical family of cutoff characteristics, where I cork is the parameter. The low-field plateau is tuned to corkscrew

resonance ( $\mathrm{I}$ low $=12.95 \mathrm{~A}$ ) with $\mathrm{V}_{0}=4 \mathrm{kV}$. The mirror ratio is $M=8.53$. 
so

$$
I_{\operatorname{coll}}=\frac{\mathrm{e}}{\mathrm{m}} \int_{\mathrm{u}_{\text {ret }}}^{\infty} \mathrm{F}\left(\mathrm{V}_{\mid \mathrm{II}}\right) \mathrm{dV_{ \| }}
$$

where we now have changed the variable of integration to $\mathrm{V}_{11}$, the axial beam voltage (or equivalently, axial beam energy), and

$$
F\left(V_{1 I}\right) \triangleq \sqrt{\frac{2 e}{m}} f(u)
$$

From (4) and (5) we have

$$
\frac{\partial I_{\operatorname{col} 1}}{\partial V_{I I}}=\sqrt{2}\left(\frac{e}{m}\right)^{3 / 2} f(u)
$$

which is the desired relationship.

Figure 6 gives the differentiated form of the curves of Figure 5, in units of $\mu \mathrm{A} / \mathrm{kV}$, and from Eqs. (5) and (6) these curves correspond to either the axial-velocity or the axialenergy distribution, within a scaling factor. We shall now attempt to correlate these results with the theory.

In a series of papers following Wingerson's original description of the magnetic corkscrew, both he and other workers (refs. 5,6 , and 7) have indicated the existence of a range of favorable entrance angles, such that when an (already spiraling) electron enters the corkscrew field with a phase angle within this range, this electron will in effect be locked to the phase-space trajectory of a perfectly synchronous electron during its entire transit through the corkscrew. The non-synchronous electron will, however, execute oscillations of increasing amplitude about a mean value of phase, and oscillations of roughly constant amplitude about a mean value of energy. This latter feature is rather important, for it implies an energy distribution of constant width, independent of the degree of conversion. This is illustrated in Figure 7, where the normalized axial energy is calculated as a function of distance both for an electron in perfect synchronism and for an electron that is assumed to suffer a $15^{\circ}$ perturbation from perfect synchronism at an axial position close to the entrance plane.

However, the prediction of a constant-width energy distribution is not borne out by the results plotted in Figure 6 . One might be tempted to ascribe this to the fact that the corkscrew 


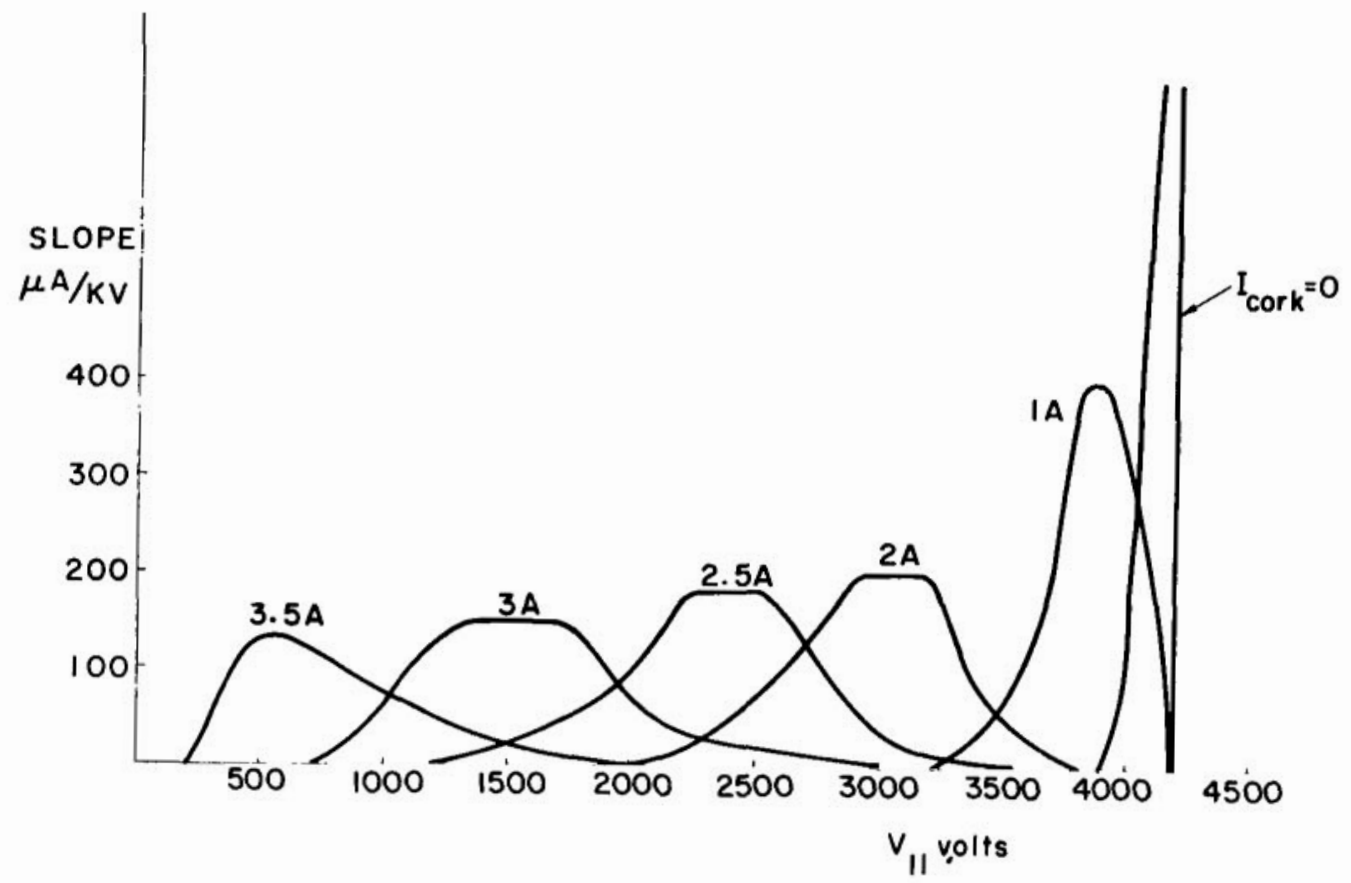

Figure 6. Axial velocity distribution corresponding to the cutoff characteristics of Figure 5 . The parameter is I cork.

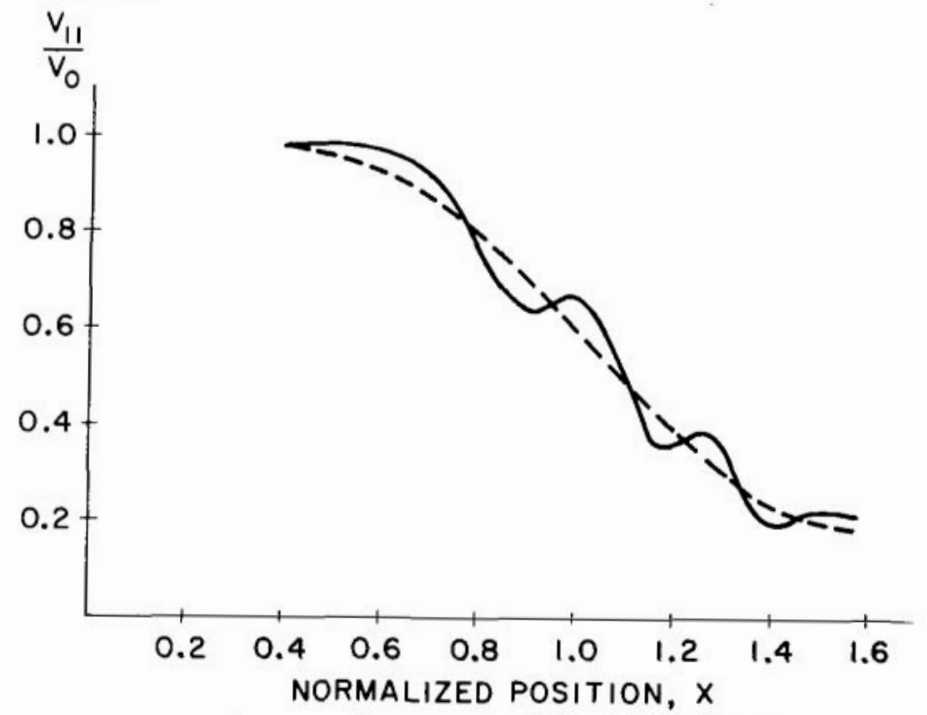

Figure 7. A computer plot of normalized axial energy vs normalized axial position along the corkscrew (after ref. 6). $x=\frac{\pi Z}{2 L}$ where $Z$ is the position of the electron, and $L$ is the length of the corkscrew. Two electron trajectories are shown, one of which is unperturbed, i.e., in perfect resonance (dashed ijne) and the other has an angular perturbation of $15^{\circ}$ at $x=0.4$ (solid line). 
had a constant pitch rather than a tapered pitch. As the synchronism condition is

$$
p(z)=\frac{2 \pi}{\eta B_{O}} u(z)
$$

we have, for constant pitch,

$$
B_{O}(z)=\frac{2 \pi}{n p} u(z)
$$

and certainly one would expect to improve the overall resonance condition by decreasing $\mathrm{B}_{\mathrm{O}}$, corresponding to a monotonic decrease of $u(z)$ along the corkscrew.

However, an increase in $B_{0}$ gives the desired improvement in the energy distribution, whereas a decrease does the opposite. This is shown in Figure 8 , where the low field has been varied in either direction about the measured cyclotron resonance. Figure $8 \mathrm{~b}$ does closely approximate the predicted constant-width energy distribution, independent of $I_{\text {cork }}$. The low field here actually was set at 406G, i.e., higher than both the measured and the design resonance values. The observed monotonic narrowing of the energy distribution with increasing low field is probably a variation of the "locking" mechanism illustrated in Figure 7, that is, tuning above resonance results in further narrowing of the "lock-in" range of energy until synchronism can no longer be maintained.

During these measurements, it was discovered also that the corkscrew current itself can cause a quasi-resonant behavior in the target current, depending on the tuning of the low-field plateau. An example is given in Figure 9, which shows that when the beam electrons are tuned above cyclotron resonance, there may be one value of corkscrew current which gives the maximum energy conversion, while values both below and above it result in less efficient corkscrew action. This phenomenon is not understood. Evidently, the mechanical simplicity of the corkscrew hides great physical complexity, and more effort can usefully be spent on understanding this fascinating device.

\section{Resolution of Corkscrew and Ramp Effects}

A further series of measurements was run with the object of separating the contributions of the corkscrew and of the magnetic ramp to the mean transverse energy and to the energy spread. This was done by measuring the energy distribution, with $I_{\text {cork }}$ as 


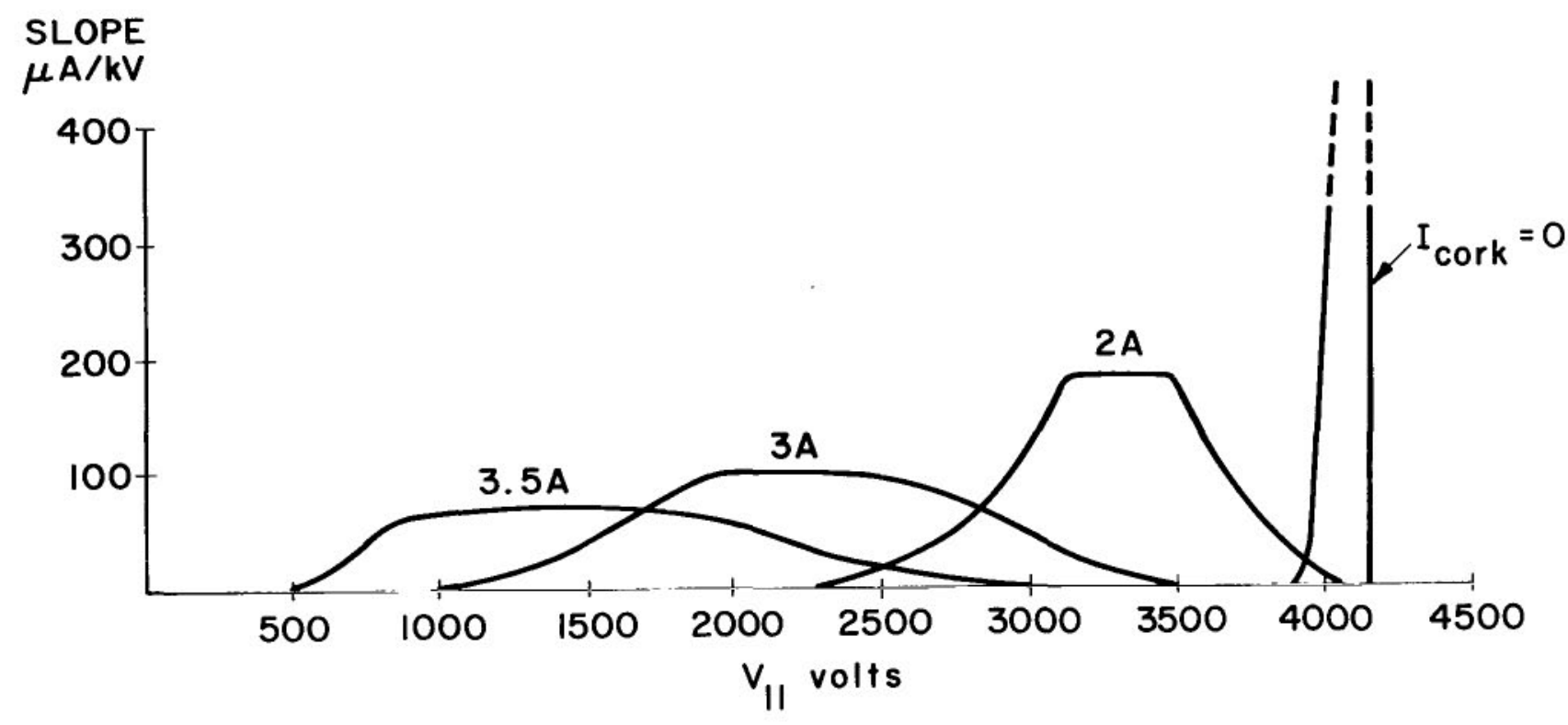

(a)

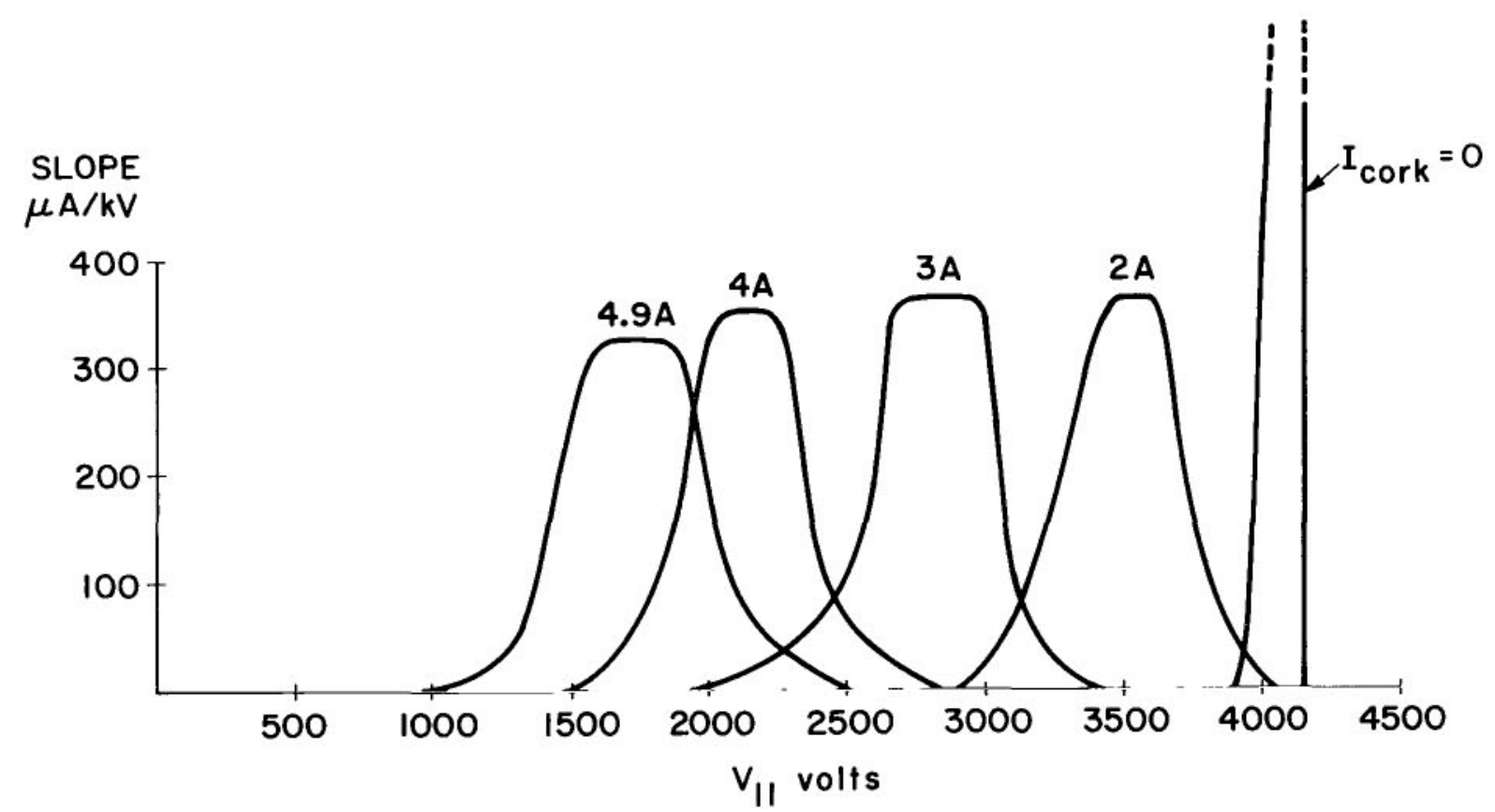

(b)

Figure 8. Energy distributions for the beam of Figure 6 , but with the electrons tuned a) below and b) above cyclotron resonance. The parameter is $I_{\text {cork }} ; V_{0}=4 \mathrm{kV}$ and $M=8.53$. Cyclotron resonance occurs at I low $=12.95 \mathrm{~A}$, and the low field is detuned to $(a) 12.3 A$ and (b) $13.4 A$. 
a parameter, for $M=1.70,4.27$, and 8.53 with $\mathrm{V}_{\mathrm{o}}=4 \mathrm{kV}$ and for $M=6.05$ with $V_{0}=2 \mathrm{kV}$.

In order to minimize the energy spread throughout this series of measurements, the low-field plateau was detuned arbitrarily by 2.5 percent above the measured cyclotron resonance value. This corresponds almost exactly to the design resonance. A typical family of cutoff characteristics is shown in Figure 10. Note that as in Figure $8 \mathrm{~b}$, the energy distribution essentially retains both its shape and its width over a wide range of the corkscrew current. Two parameters of interest are the energy spread $\Delta V$ and the mean transverse energy $\left\langle\mathrm{V}_{\perp}\right\rangle$. The latter we shall define as the difference between the initial beam voltage and the mean value of the axial energy distribution

$$
\left\langle\mathrm{v}_{\perp}\right\rangle \triangleq \mathrm{v}_{0}-\left\langle\mathrm{v}_{11}\right\rangle
$$

Assuming the energy conversion in the magnetic ramp to be adiabatic so that the magnetic moment $\frac{\mathrm{eV}_{\perp}}{\mathrm{B}}$ is conserved, the transverse energy will increase linearly with the axial magnetic field, i.e., the mirror ratio $M$. Thus one would expect $\frac{1}{M}\left\langle v_{\perp}\right\rangle$ to be constant for a given $I_{\text {cork }}$. The amount of energy conversion achieved in the corkscrew region is related to the beam and the corkscrew geometry by a fixed constant, and the expected dependence on $I_{\text {cork }}$ may be deduced from a simple argument, as follows.

Since the transverse electron velocity $\mathrm{v}$ can be assumed to be primarily orbital in direction, the axial component of the equation of motion may be approximated by

$$
\frac{d v}{d z} \cong-\frac{e}{m} B_{r}=\text { constant }
$$

where $B_{r}$ is the radial component of the corkscrew field (ref. 5). Thus, over an arbitrary length $\Delta \mathbf{z}$ the change in transverse momentum will be

$$
\Delta \mathrm{v} \cong-\frac{\mathrm{e}}{\mathrm{m}} \mathrm{B}_{\mathrm{r}} \Delta \mathrm{z} \sim \mathrm{I}_{\mathrm{cork}} \Delta \mathrm{z}
$$




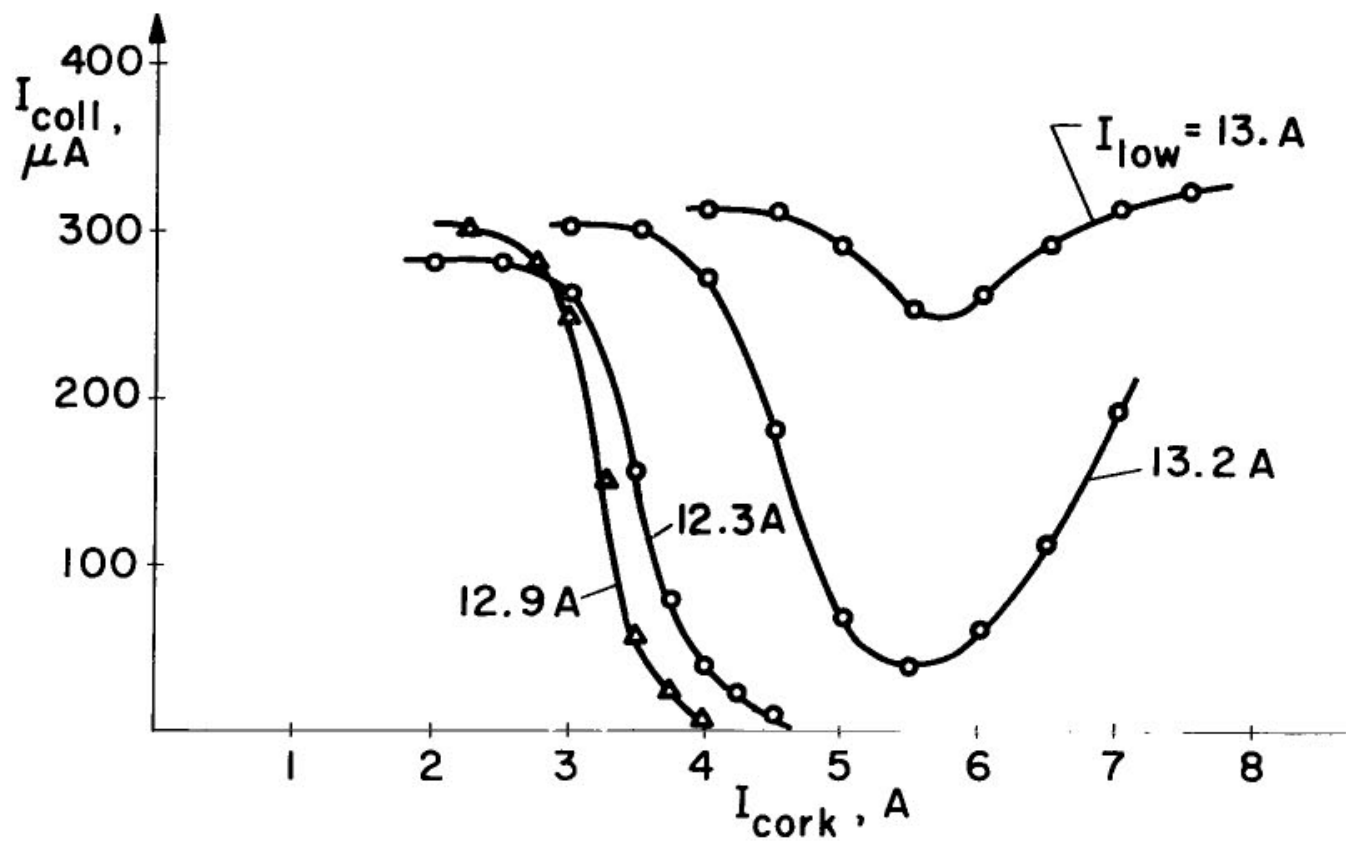

Figure 9. A plot of target current vs corkscrew current, with low field current as the parameter. Cyclotron-resonance tuning corresponds to $\mathrm{I}_{\mathrm{low}}=12.9 \mathrm{~A}$. The retarding lens was set at $\mathrm{V}_{\text {ret }}=900^{\circ} \mathrm{V}$, with $I_{\text {coll }, \mu \mathrm{A}} \quad V_{0}=4 \mathrm{kV}$ and $M=8.53$.
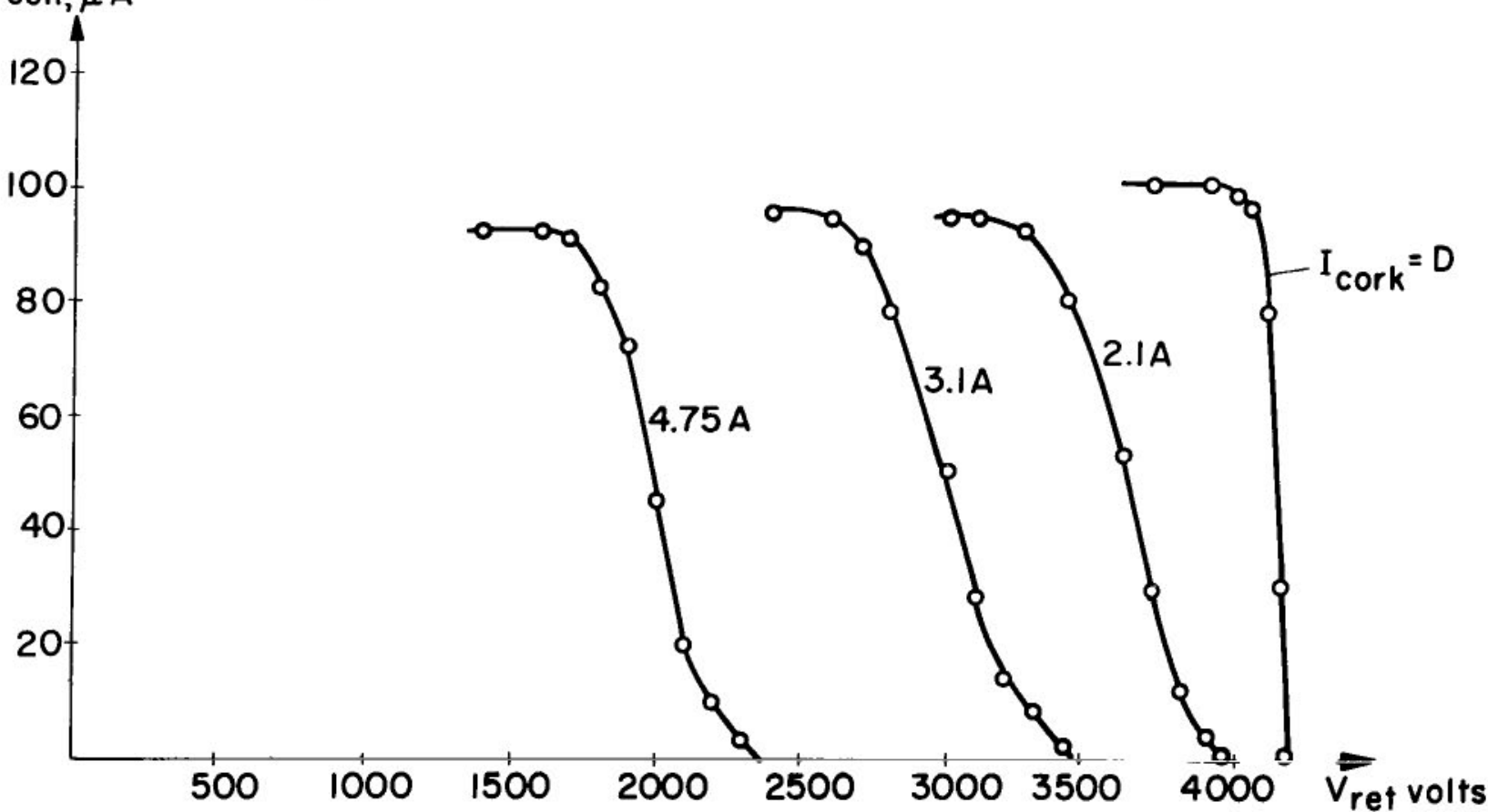

Figure 10. Typical cutoff characteristics when the low field is tuned to the design cyclotron resonance. The parameter is I cork, and $V_{0}=4 \mathrm{kV}, M=4.27$. 
But since $\mathrm{v}=0$ at $\mathrm{z}=0, \mathrm{v}=\Delta \mathrm{v}$, and we can also write the transverse energy

$$
\mathrm{V}_{\perp} \sim \mathrm{v}_{\perp}^{2} \sim \mathrm{I}_{\mathrm{cork}}^{2}
$$

which is the desired relationship. Incidentally, Eq. (8) also provides an approximate prescription for tapering the corkscrew.

An experimental check of these conclusions is provided by Figure 11, where the equation

$$
\frac{1}{\bar{M}}\left\langle\mathrm{~V}_{\perp}\right\rangle=\mathrm{KI}_{\text {cork }}^{2}, \quad \mathrm{~K}=29.9
$$

is seen to provide a close fit to the measured data.

It remains to explore the dependence of the energy spread on the mirror ratio, for a given amount of corkscrew modulation. If, as before, one assumes the energy conversion in the ramp region to be adiabatic, then the transverse energy at the output will follow the transverse energy at the input linearly,

$$
\mathrm{V}_{\perp, 2}=\mathrm{M} \mathrm{V}_{\perp, 1}
$$

The same law holds over a range of energy:

$$
\mathrm{v}_{\perp, 2}+\Delta \mathrm{v}_{\perp, 2}=\mathrm{M}\left(\mathrm{v}_{\perp, 1}+\Delta \mathrm{v}_{\perp, 1}\right)
$$

and subtracting (12) from (13) gives the desired equation,

$$
\Delta \mathrm{V}_{\perp, 2}=\mathrm{M} \Delta \mathrm{V}_{\perp}, 1
$$

that is, the magnetic ramp will amplify the energy spread linearly.

Eq. (14) is essentially confirmed by the results shown in Figure 12, where the measured energy spread, normalized to the beam voltage $V_{o}$, is plotted vs the mirror ratio. The scatter of data points reflects the non-ideal behavior of the corkscrew action as $I_{\text {cork }}$ is changed, for if the corkscrew behaved ideally, the energy spread due to it would be nearly constant, independent

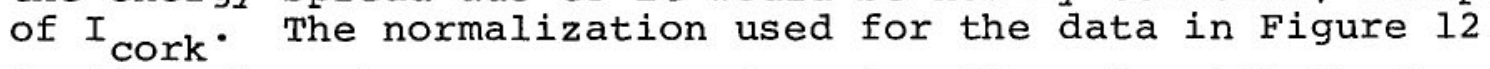

implies that the energy spread scales linearly with the beam voltage $\mathrm{V}_{0}$. While this may be an intuitively reasonable assumption, it was also tested experimentally, with $\mathrm{v}_{0}=2,4$, and $6 \mathrm{kV}$. 


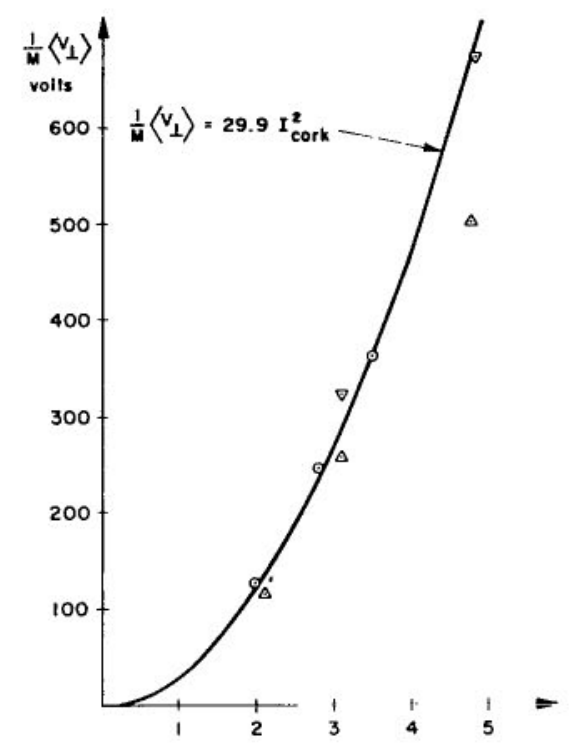

Figure 11. A plot of the mean transverse energy, normalized to the mirror ratio, versus the corkscrew current. The data are derived from measurements with $V_{0}=4 \mathrm{kV}$ and $M=1.70(\Delta), M=4.27(\Delta)$, and $M^{0}=8.53(\odot)$.

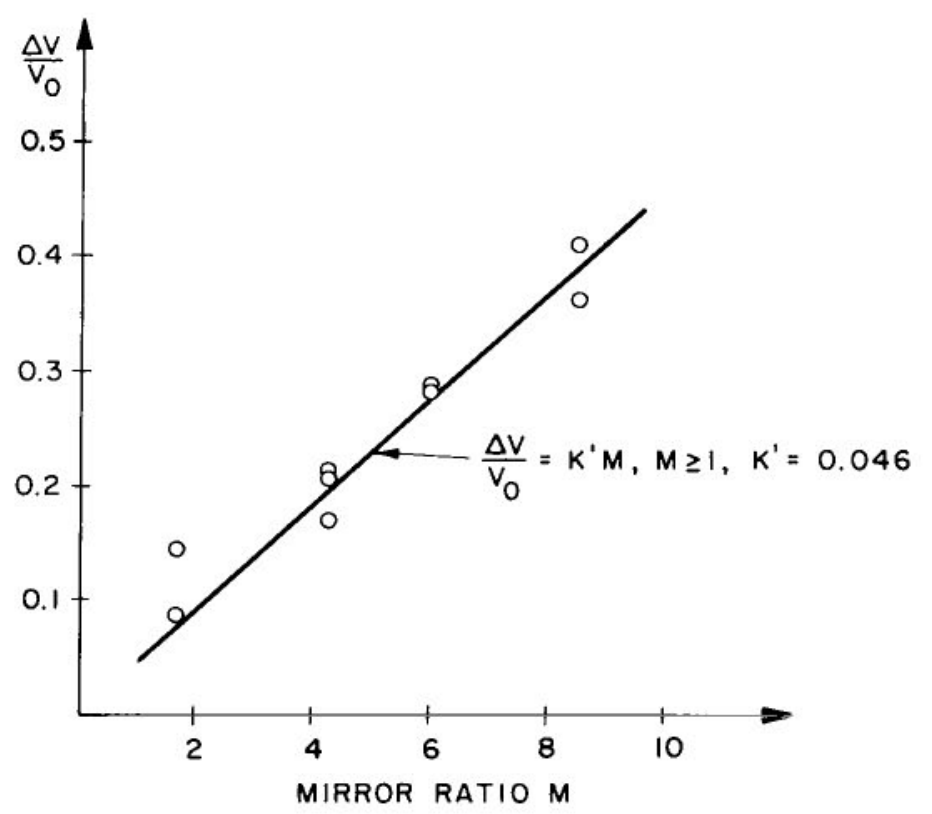

Figure 12. Normalized energy spread plotted vs mirror ratio $M$. For each value of $M$, several points are plotted, corresponding to different values of corkscrew current for that particular beam. Data are plotted for a $2 \mathrm{kV}$ beam with $\mathrm{M}=6.05$, and for a $4 \mathrm{kV}$ beam with $M=1.70,4.27,8.53$. 
The corkscrew current was adjusted to give $\langle V\rangle \simeq 0.25 \mathrm{~V}$, for each beam. The results (Fig. 13) confirm that indeed $\Delta V \sim V_{0}$ as stated.

\section{A Method of Energy Analysis Using Beam Mirroring}

For practical beam design, it would be desirable to be able to calculate quickly the constant K in Eq. (11) and the coefficient of $M$ in Figure 12, as this would define the conversion efficiency and the energy spread associated with any particular corkscrew. Unfortunately, such calculations are difficult and tedious, because the corkscrew action is physically quite complex. An empirical calibration is perhaps the next-best solution, and some tests were made to see whether this could be done without recourse to a retarding lens arrangement, i.e., by using the mirroring action of the magnetic ramp itself to estimate the energy spectrum of the beam. Such a procedure can indeed be developed, based only on the assumption that the energy distribution is symmetrical about some point, which generally seems to be the case. The method relies on target current cutoff characteristics of the type shown in Figure 9, with the low-field plateau adjusted for cyclotron resonance, and it is explained in Figure 14. For simplicity, a rectangular energy distribution is assumed, although this is not essential to the method. At the three successive values of corkscrew current shown, the collector current will begin to decrease due to mirroring at (a), will have dropped to one-half its maximum value at (b), and will have vanished at (c). The shift of part of the energy distribution into the $\left\langle V_{11}\right\rangle<0$ region indicates mirroring of the corresponding fraction of the beam. We note immediately that in (b) we have

$\left\langle V_{\perp}\right\rangle=V_{0}$, and this therefore defines $\mathrm{K}$ in Eq. (11), or

$$
K=\frac{V_{0}}{I_{2}^{2}}
$$

Also, by combining the information from (a) and (c) we find

$$
\begin{aligned}
\mathrm{K}\left(\mathrm{I}_{3}^{2}-\mathrm{I}_{1}^{2}\right) & =\mathrm{V}_{0}-\left\langle\mathrm{V}_{0,3}\right\rangle-\mathrm{V}_{0}+\left\langle\mathrm{V}_{0,1}\right\rangle \\
& =\left\langle\mathrm{V}_{0,1}\right\rangle-\left\langle\mathrm{V}_{0,3}\right\rangle \\
& =\Delta \mathrm{V}
\end{aligned}
$$




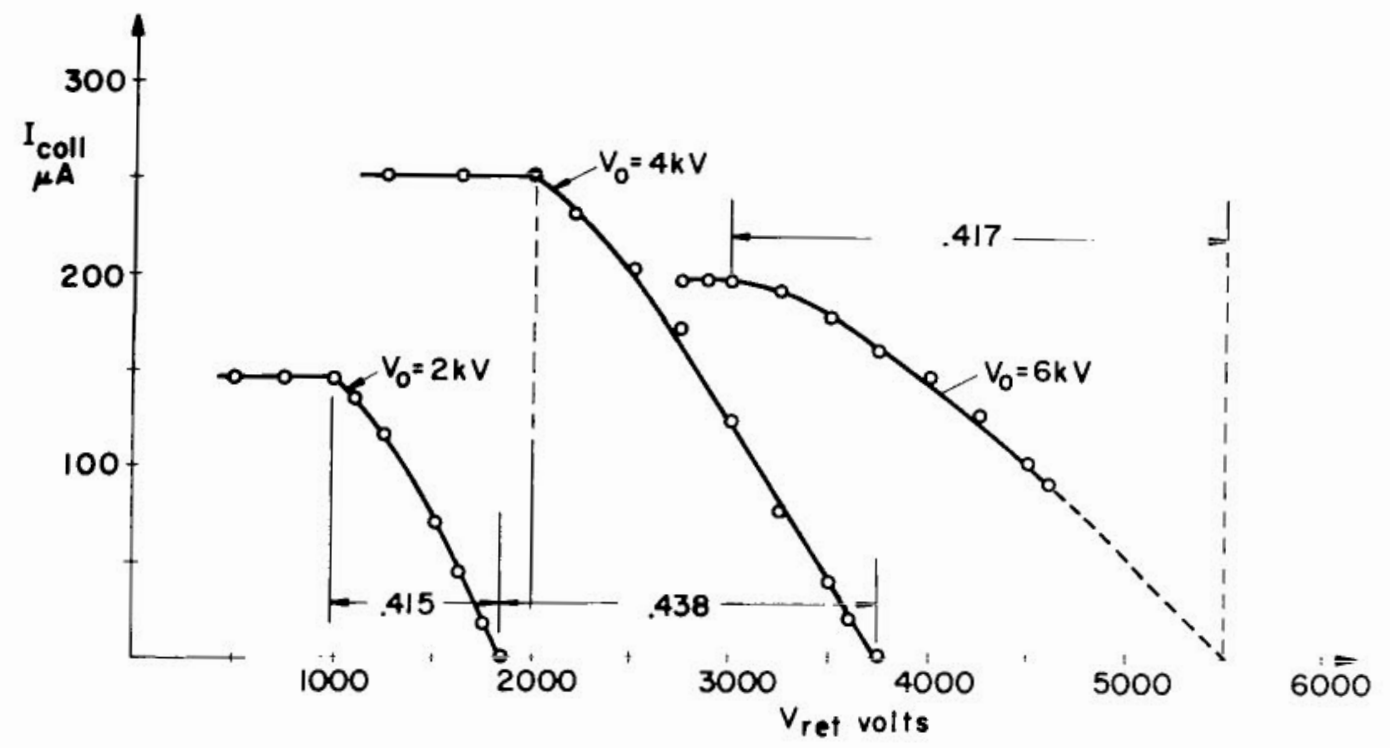

Figure 13. Cutoff characteristics with beams of different $V_{0}$. For each beam, the corkscrew current is adjusted ${ }^{\circ}$ to give $\left\langle V_{\perp}\right\rangle \simeq 0.25 V_{0}$. The mirror ratio is $M=8.53$, and the low-field plateau is tuned to cyclotron resonance. The measured values of $\frac{\Delta V}{V_{0}}$ are indicated on each curve. The beam current was controlled by temperature-limiting the cathode.
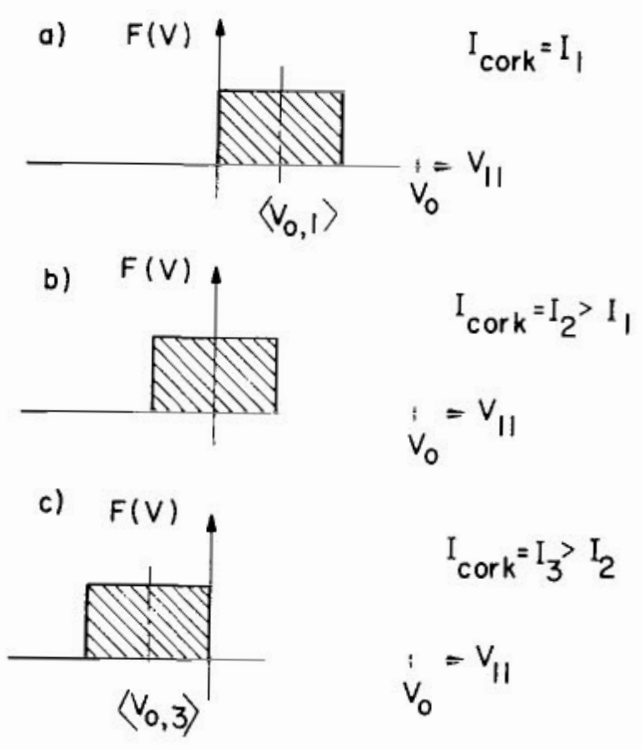

$$
\begin{aligned}
& I_{\text {cork }}=I_{3}>I_{2} \\
& V_{0}=V_{11}
\end{aligned}
$$

Figure 14. A method of estimating the corkscrew efficiency and the energy spread. The idealized energy distribution gradually shifts into the mirroring region $V_{11}<0$ as the corkscrew current is increased from $\mathrm{I}_{1}$ to I 3 . The collector current decreases correspondingly. 
and combining (15) and (16) the energy spread is

$$
\frac{\Delta V}{V_{0}}=\frac{I_{3}{ }^{2}-I_{1}{ }^{2}}{I_{2}{ }^{2}}
$$

This method was applied to the beams of Figure 13, where, by using the retarding-field analyzer, the energy spreads previously had been measured as $0.415,0.438$, and 0.417 , respectively. The results obtained by the mirxoring method were $0.527,0.410$, and 0.485 , respectively, and thus the two methods agree within better than 20 percent.

\section{CONCLUSION}

The measurements described in this report indicate that in the absence of space-charge effects, a relatively narrow, nearrectangular energy distribution will be generated by the corkscrew when the axial magnetic field is tuned to cyclotron resonance. The normalized width of this distribution is independent of the degree of energy conversion, in agreement with the theory, and the mean transverse (converted) energy varies as the square of the corkscrew current. The magnetic ramp amplifies both the mean transverse energy and the energy spread linearly, as expected from the assumed adiabatic conversion. It is shown that both quantities also can be estimated by using the mirroring effect of the magnetic ramp, in conjunction with variable corkscrew current. 
1. Kulke, B.: Design Considerations for Cyclotron Resonance Oscillators. NASA TN D-5237, June, 1969.

2. Wingerson, R.: Corkscrew - A Device for Changing the Magnetic Moment of Charged Particles in a Magnetic Field. Phys. Rev. Letters, vol. 6, May 1, 1961, pp. 446-448.

3. Kulke, B., and Wilmarth, R.W.: Small-Signal and Saturation Characteristics of an X-band Cyclotron - Resonance Maser. Proc. IEEE, (letters), vol. 57, February 1969, pp. 219-220.

4. Caulton, M.: Retarding-field Analyzers for the Measurement of Axial-Velocity Distributions in Electron Beams. RCA Review, vol. 26, June 1965, pp. 217-24l.

5. Wingerson, R. C., Dupree, T. H., and Rose, D. J.: Trapping and Loss of Charged Particles in a Perturbed Magnetic Field. Physics of Fluids, vol. 7, no. 9, September 1964, pp. $1475-1484$.

6. Lidsky, L.M.: Orbit Stability in a Helically Perturbed Magnetic Field. Phys. Fluids, vol. 7, September 1964, pp. $1484-1488$.

7. Dreicer, H., et al.: Cyclotron Resonance in the Static Magnetic Field of a Helix. Nuclear Fusion, 1962 Supplement, Part 1, pp. 299-312 (Proceedings of the Conference on Plasma Physics and Controlled Nuclear Fusion Research, 4-9 September 1961, Salzburg, Austria). 


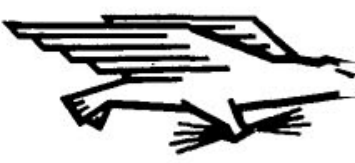

POSTAGE AND FEES PAII NATIONAL AERONAUTICS SPACE ADMINISTRATION

"The aeronatical and space activities of the United States shall be conducted so as to contribute. . . to the expansion of buman knowledge of phenomena in the atmosphere and space. The Administration shall protide for the widest practicable and appropriate dissemination of information concerning its activities and the results thereof."

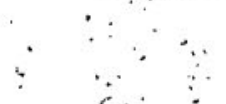

- National Aeronautics And Space ACt Of 1958

\section{NASA SCIENTIFIC AND TECHNICAL PUBLICATIONS}

TECHNICAL REPORTS: Scientific and technical information considered important, complete, and a lasting contribution to existing knowledge.

TECHNICAL NOTES: Information less broad in scope but nevertheless of importance as a contribution to existing knowledge.

TECHNICAL MEMORANDUMS:

Information receiving limited distribution because of prelimimary.data, security classification, or other réăsons. '

CONTRACTOR REPORTS: Scientific and technical information generated under a NASA contract or grant and considered an important contribution to existing knowledge.
TECHNICAL TRANSLATIONS: Information published in a foreign language considered to merit NASA distribution in English.

SPECIAL PUBLICATIONS: Information derived from or of value to NASA activities. Publications include conference proceedings, monographs, data compilations, handbooks, sourcebooks, and special bibliographies.

\section{TECHNOLOGY UTILIZATION}

PUBLICATIONS: Information on technology used by NASA that may be of particular interest in commercial and other non-acrospace applications. Publications include Tech Briefs, Technology Utilization Reports and Notes, and Technology Surveys.

Details on the availability of these publications may be obtained from:

SCIENTIFIC AND TECHNICAL INFORMATION DIVISION

NATIONAL AERONAUTICS AND SPACE ADMINISTRATION

Washington, D.C. 20546 